1 Hacettepe Journal of Mathematics and Statistics

$\bigcap$ Volume 46 (3) (2017), 427-435

\title{
On some new length problem for analytic functions
}

\author{
Janusz Sokół* and Mamoru Nunokawa ${ }^{\dagger}$
}

\begin{abstract}
Let $\mathcal{H}$ denote the class of analytic functions in the unit disk $|z|<1$. Let $C(r, f)$ be the closed curve which is the image of the circle $|z|=r<1$ under the mapping $w=f(z) \in \mathcal{H}, L(r, f)$ the length of $C(r, f)$ and let $A(r, f)$ be the area enclosed by $C(r, f)$. Let $l\left(r e^{i \theta}, f\right)$ be the length of the image curve of the line segment joining $r e^{i \theta}$ and $r e^{i(\theta+\pi)}$ under the mapping $w=f(z)$ and let $l(r, f)=\max _{0 \leq \theta<2 \pi} l\left(r e^{i \theta}, f\right)$. We find upper bound for $l(r, f)$ for $f(z)$ in some known classes of functions. Moreover, we prove that $l(r, f)=\mathcal{O}\left(\log \frac{1}{1-r}\right)$ and that $L(r, f)=\mathcal{O}\left\{A(r, f) \log \frac{1}{1-r}\right\}^{1 / 2}$ as $r \rightarrow 1$ under weaker assumptions on $f(z)$ than some previous results of this type.
\end{abstract}

Keywords: length problem ; convex functions; starlike functions 2000 AMS Classification: Primary 30C45, Secondary 30C80

Received : 22.01.2016 Accepted : 14.08.2016 Doi : 10.15672/HJMS.20174620773

\section{Introduction}

Let $\mathcal{H}$ denote the class of analytic functions in the unit disk $\mathbb{D}=\{z \in \mathbb{C}:|z|<1\}$. Let $\mathcal{A}$ be the class of functions

$$
f(z)=z+\sum_{n=2}^{\infty} a_{n} z^{n}
$$

which are analytic in $\mathbb{D}$. Recall that a set $E \subset \mathbb{C}$ is said to be starlike with respect to a point $w_{0} \in E$ if and only if the linear segment joining $w_{0}$ to every other point $w \in E$ lies entirely in $E$, while a set $E$ is said to be convex if and only if it is starlike with respect to each of its points, that is if and only if the linear segment joining any two points of $E$

*Faculty of Mathematics and Natural Sciences, University of Rzeszów, ul. Prof. Pigonia 1, 35-310 Rzeszów, Poland

Email : jsokol@ur.edu.pl

$\dagger$ University of Gunma, Hoshikuki-cho 798-8, Chuou-Ward, Chiba, 260-0808, Japan

Email : mamoru_nuno@doctor.nifty.jp 
lies entirely in $E$. A univalent function $f$ maps $\mathbb{D}$ onto convex domain $E$ if and only if [13]

$$
\mathfrak{R e}\left\{1+\frac{z f^{\prime \prime}(z)}{f^{\prime}(z)}\right\}>0 \quad \text { for all } z \in \mathbb{D}
$$

and then $f(z)$ is said to be convex in $\mathbb{D}$ (or briefly convex). Let $\mathcal{S}$, $\mathcal{C}$ denote the subclasses of $\mathcal{A}$ consisting of all functions that are univalent, convex univalent in $\mathbb{D}$, respectively.

Let $C(r, f)$ be the closed curve which is the image of $|z|=r<1$ under the mapping $w=f(z)$. Let $L(r, f)$ denote the length of $C(r, f)$ and let $A(r, f)$ be the area enclosed by $C(r, f)$.

If $f \in \mathcal{A}$ satisfies

$$
\mathfrak{R e}\left\{\frac{z f^{\prime}(z)}{f(z)}\right\}>0, z \in \mathbb{D},
$$

then $f(z)$ is said to be starlike with respect to the origin in $\mathbb{D}$ and it is denoted by $f(z) \in \mathcal{S}^{*}$. It is known that $\mathcal{S}^{*} \subset \mathcal{S}$.

Definition. Let $l\left(r e^{i \theta}, f\right)$ be the length of the image curve of the line segment joining $r e^{i \theta}$ and $r e^{i(\theta+\pi)}$ under the mapping $w=f(z), f(z) \in \mathcal{H}$

$$
l\left(r e^{i \theta}, f\right)=\int_{-r}^{r}\left|f^{\prime}\left(\rho e^{i \theta}\right)\right| \mathrm{d} \rho
$$

and let

$$
l(r, f)=\max _{0 \leq \theta<2 \pi} l\left(r e^{i \theta}, f\right)=\max \left\{\int_{-r}^{r}\left|f^{\prime}\left(\rho e^{i \theta}\right)\right| \mathrm{d} \rho: 0 \leq \theta<2 \pi\right\} .
$$

For instance, it is easy to establish that $l(r, z)=2 r, l\left(r, z^{2}\right)=2 r^{2}$. To find an example for bounded function recall the following result from [6]. If $f(z) \in \mathcal{A}$ then

$$
|f(z)|<1 \quad \Rightarrow \quad\left|f^{\prime}(z)\right| \leq \frac{25}{24} \quad \text { in } \quad|z| \leq \frac{1}{2}
$$

Hence, if $f(z) \in \mathcal{A},|f(z)|<1$, then $l(1 / 2, f) \leq \frac{25}{24}$. It is possible to establish $l(r, f)$ in known classes of functions. We put now suitable definition.

Let $\mathcal{Q}$ be a subclass of $\mathcal{H}$ and let $0<r<1$. We write

$$
l(r, \mathcal{Q}) \leq q(r) \quad \Leftrightarrow \quad\{\forall f \in \mathcal{Q} \forall r \quad: \quad l(r, f) \leq q(r)\}
$$

and

$$
l(r, \mathcal{Q})=s(r) \quad \Leftrightarrow \quad\{l(r, \mathcal{Q}) \leq s(r) \quad \text { and } \quad \forall r \exists g \in \mathcal{Q} \quad: \quad l(r, g)=s(r)\} .
$$

1.1. Theorem. We have

$$
l(r, \mathcal{C})=\frac{2 r}{1-r^{2}}, \quad l(r, \mathcal{S})=\frac{2 r\left(1+r^{2}\right)}{\left(1-r^{2}\right)^{2}}, \quad l\left(r, \mathcal{S}^{*}\right)=\frac{2 r\left(1+r^{2}\right)}{\left(1-r^{2}\right)^{2}},
$$

where $\mathcal{S}, \mathcal{S}^{*}, \mathcal{C}$ denote the subclasses of $\mathcal{A}$ consisting of all functions that are univalent, starlike univalent, convex univalent in $\mathbb{D}$, respectively.

Proof. Recall that if $f(z) \in \mathcal{C},[3]$, then we have

$$
\left|f^{\prime}(z)\right| \leq \frac{1}{(1-r)^{2}}, \quad z=r e^{i \theta}
$$


Therefore, for $f(z) \in \mathcal{C}$, we have

$$
\begin{aligned}
l(r, f(z)) & =\max \left\{\int_{-r}^{r}\left|f^{\prime}\left(\rho e^{i \theta}\right)\right| \mathrm{d} \rho: 0 \leq \theta<2 \pi\right\} \\
& \leq \max \left\{\int_{-r}^{r} \frac{1}{(1-\rho)^{2}} \mathrm{~d} \rho: 0 \leq \theta<2 \pi\right\} \\
& =\int_{-r}^{r} \frac{1}{(1-\rho)^{2}} \mathrm{~d} \rho \\
& =\frac{2 r}{1-r^{2}} .
\end{aligned}
$$

Thus $l(r, \mathcal{C}) \leq \frac{2 r}{1-r^{2}}$. Furthermore, $g(z)=\frac{z}{1-z}$ is in $\mathcal{C}$ and $g^{\prime}(z)=\frac{1}{(1-z)^{2}}$, hence

$$
\begin{aligned}
l\left(r, \frac{z}{1-z}\right) & =\max \left\{\int_{-r}^{r}\left|\frac{1}{\left(1-\rho e^{i \theta}\right)^{2}}\right| \mathrm{d} \rho: 0 \leq \theta<2 \pi\right\} \\
& =\max \left\{\int_{-r}^{r} \frac{1}{1+\rho^{2}-2 \rho \cos \theta} \mathrm{d} \rho: 0 \leq \theta<2 \pi\right\} \\
& =\int_{-r}^{r} \frac{1}{1+\rho^{2}-2 \rho} \mathrm{d} \rho \\
& =\frac{2 r}{1-r^{2}}
\end{aligned}
$$

This completes the proof of the first formula of (1.3). To prove the second one recall that if $f(z) \in \mathcal{S},[3]$, then we have

$$
\left|f^{\prime}(z)\right| \leq \frac{1+r}{(1-r)^{3}}, \quad z=r e^{i \theta}
$$

Therefore, if $f(z) \in \mathcal{S}$, we have

$$
\begin{aligned}
l(r, f(z)) & =\max \left\{\int_{-r}^{r}\left|f^{\prime}\left(\rho e^{i \theta}\right)\right| \mathrm{d} \rho: 0 \leq \theta<2 \pi\right\} \\
& \leq \max \left\{\int_{-r}^{r} \frac{1+\rho}{(1-\rho)^{3}} \mathrm{~d} \rho: 0 \leq \theta<2 \pi\right\} \\
& =\int_{-r}^{r} \frac{1+\rho}{(1-\rho)^{3}} \mathrm{~d} \rho \\
& =\left[\frac{\rho}{(1-\rho)^{2}}\right]_{-r}^{r} \\
& =\frac{2 r\left(1+r^{2}\right)}{\left(1-r^{2}\right)^{2}}
\end{aligned}
$$


Thus $l(r, \mathcal{S}) \leq \frac{2 r\left(1+r^{2}\right)}{\left(1-r^{2}\right)^{2}}$. Furthermore, consider the Koebe function $f(z)=\frac{z}{(1-z)^{2}}$, then $f^{\prime}(z)=\frac{1+z}{(1-z)^{3}}$, hence

$$
\begin{aligned}
l\left(r, \frac{z}{(1-z)^{2}}\right) & =\max \left\{\int_{-r}^{r}\left|\frac{1+\rho e^{i \theta}}{\left(1-\rho e^{i \theta}\right)^{3}}\right| \mathrm{d} \rho: 0 \leq \theta<2 \pi\right\} \\
& =\max \left\{\int_{-r}^{r} \frac{\sqrt{1+\rho^{2}+2 \rho \cos \theta}}{\left(1+\rho^{2}-2 \rho \cos \theta\right)^{3 / 2}} \mathrm{~d} \rho: 0 \leq \theta<2 \pi\right\} \\
& =\int_{-r}^{r} \frac{\sqrt{1+\rho^{2}+2 \rho}}{\left(1+\rho^{2}-2 \rho\right)^{3 / 2}} \mathrm{~d} \rho \\
& =\int_{-r}^{r} \frac{1+\rho}{(1-\rho)^{3}} \mathrm{~d} \rho \\
& =\left[\frac{\rho}{(1-\rho)^{2}}\right]_{-r}^{r} \\
& =\frac{2 r\left(1+r^{2}\right)}{\left(1-r^{2}\right)^{2}} .
\end{aligned}
$$

This shows that $l(r, \mathcal{S})=\frac{2 r\left(1+r^{2}\right)}{\left(1-r^{2}\right)^{2}}$. Because the Koebe function is also starlike function then $l\left(r, \mathcal{S}^{*}\right)=\frac{2 r\left(1+r^{2}\right)}{\left(1-r^{2}\right)^{2}}$ too.

In [7] it was introduced the following class

$$
\mathcal{N}^{\prime}=\left\{f(z) \in \mathcal{A}:\left|\arg \left\{f^{\prime}(z)\right\}\right|<\pi\right\} .
$$

It was proved in [7] that if $f(z) \in \mathcal{N}^{\prime}$, then

$$
\left|f^{\prime}(z)\right| \leq\left(\frac{1+r}{1-r}\right)^{2} \quad, \quad z=r e^{i \theta} .
$$

Therefore, for $f(z) \in \mathcal{N}^{\prime}$, we have

$$
\begin{aligned}
l(r, f(z)) & =\max \left\{\int_{-r}^{r}\left|f^{\prime}\left(\rho e^{i \theta}\right)\right| \mathrm{d} \rho: 0 \leq \theta<2 \pi\right\} \\
& \leq \max \left\{\int_{-r}^{r}\left(\frac{1+\rho}{1-\rho}\right)^{2} \mathrm{~d} \rho: 0 \leq \theta<2 \pi\right\} \\
& =\left[\rho+4 \log (1-\rho)+\frac{4}{1-\rho}\right]_{-r}^{r} \\
& =2 r+4 \log \frac{1-r}{1+r}+\frac{8 r}{1-r^{2}} .
\end{aligned}
$$

Thus $l\left(r, \mathcal{N}^{\prime}\right) \leq 2 r+4 \log \frac{1-r}{1+r}+\frac{8 r}{1-r^{2}}$. Furthermore, The function $h(z)=(1+z)^{2} /(1-z)^{2}$ shows that $l\left(r, \mathcal{N}^{\prime}\right)=2 r+4 \log \frac{1-r}{1+r}+\frac{8 r}{1-r^{2}}$

$$
\text { If } \begin{aligned}
& f(z)=\frac{1+z}{1-z}, \text { then } f^{\prime}(z)=\frac{2}{(1-z)^{2}}, \text { hence } \\
& \qquad \begin{aligned}
l\left(r, \frac{1+z}{1-z}\right) & =\max \left\{\int_{-r}^{r}\left|\frac{2}{\left(1-\rho e^{i \theta}\right)^{2}}\right| \mathrm{d} \rho: 0 \leq \theta<2 \pi\right\} \\
& =\max \left\{\int_{-r}^{r} \frac{2}{1+\rho^{2}-2 \rho \cos \theta} \mathrm{d} \rho: 0 \leq \theta<2 \pi\right\} \\
& =\int_{-r}^{r} \frac{2}{1+\rho^{2}-2 \rho} \mathrm{d} \rho \\
& =\frac{4 r}{1-r^{2}} .
\end{aligned}
\end{aligned}
$$


It is known that if $\mathcal{P}$ denotes the class of functions $h(z) \in \mathcal{H}, h(z)=1+\ldots$, with positive real part in the unit disc, then

$$
\left|h^{\prime}(z)\right| \leq \frac{2}{(1-r)^{2}}, \quad z=r e^{i \theta}
$$

Therefore, applying the same methods as the above we can obtain that $l(r, \mathcal{P})=\frac{4 r}{1-r^{2}}$.
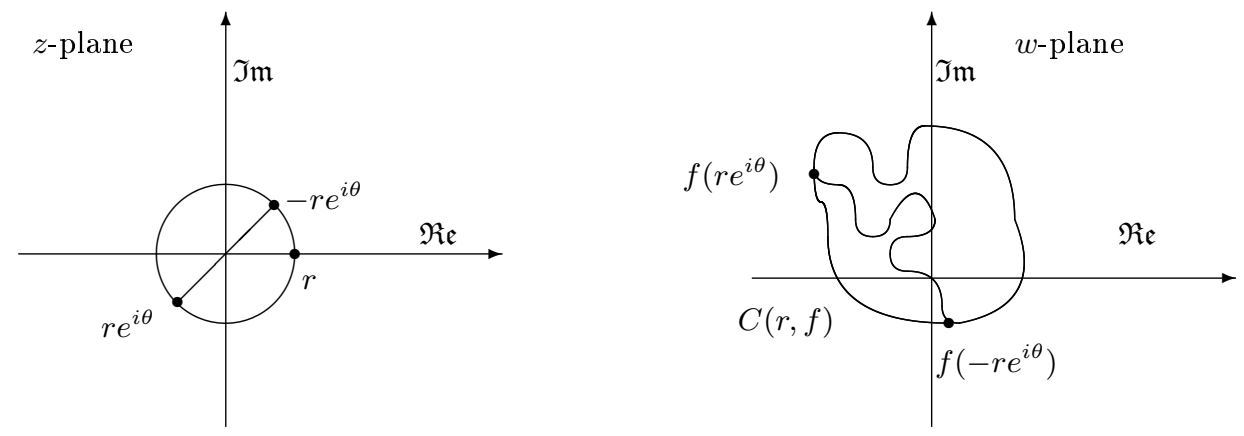

Fig. 1. A picture for Definition.

\section{Main results}

2.1. Theorem. Let $f(z)$ be analytic in $\mathbb{D}$ and suppose that $f^{\prime}(z)$ be continuous on $\overline{\mathbb{D}}$. Then we have

$$
l(r, f) \leq \frac{1}{2} L(r, f)
$$

Proof. We will need the following Fejér-Riesz's result:

2.2. Lemma. [2], [3, p.175] Let $g(z)$ be analytic in $\mathbb{D}$ and continuous on $\overline{\mathbb{D}}$ and $0<p$. Then we have

$$
\int_{-1}^{1}|g(z)|^{p} \mathrm{~d} z \leq \frac{1}{2} \int_{|z|=1}|g(z)|^{p}|\mathrm{~d} z|
$$

where the integral on the left is taken along the real axis.

We have

$$
l\left(r e^{i \theta}, f\right)=\int_{-r}^{r}\left|f^{\prime}\left(\rho e^{i \theta}\right)\right| \mathrm{d} \rho=\int_{-1}^{1}\left|f^{\prime}\left(r t e^{i \theta}\right)\right| \mathrm{d}(r t)=\int_{-1}^{1}\left|f^{\prime}(z)\right||\mathrm{d} z|
$$

for all $\theta, 0 \leq \theta<2 \pi$. Moreover,

$$
L(r, f)=\int_{0}^{2 \pi}\left|t e^{i \theta} f^{\prime}\left(t e^{i \theta}\right)\right| \mathrm{d} \theta=\int_{|z|=1}\left|f^{\prime}(z)\right||\mathrm{d} z|
$$

Applying Fejér-Riesz's lemma 2.2 for $p=1$ and $g(z)=f^{\prime}(z)$, we have

$$
\int_{-1}^{1}\left|f^{\prime}(z)\right||\mathrm{d} z| \leq \frac{1}{2} \int_{|z|=1}\left|f^{\prime}(z)\right||\mathrm{d} z|
$$


therefore, from (2.3) and from (2.4), we have

$$
l\left(r e^{i \theta}, f\right) \leq \frac{1}{2} L(r, f)
$$

for all $\theta, 0 \leq \theta<2 \pi$. This gives

$$
l(r, f)=\max _{0 \leq \theta<2 \pi} l\left(r e^{i \theta}\right) \leq \frac{1}{2} L(r, f) .
$$

2.3. Remark. Theorem 2.1 says that $l\left(r e^{i \theta}, f\right)$ may not to be too large in comparison with $L(r, f)$.

2.4. Theorem. Let $f(z)$ be analytic in $\mathbb{D}$ and suppose that

$$
\left|f^{\prime}(z)\right| \leq\left|\frac{1+z}{1-z}\right| \quad z \in \mathbb{D},
$$

then we have

$$
l(r, f) \leq \mathcal{O}\left(\log \frac{1}{1-r}\right) \quad \text { as } \quad r \rightarrow 1
$$

where $\mathcal{O}$ means Landau's symbol.

Proof. From the hypothesis (2.7) it follows that

$$
\begin{aligned}
l\left(r e^{i \theta}, f\right) & =\int_{-r}^{r}\left|f^{\prime}\left(\rho e^{i \theta}\right)\right| \mathrm{d} \rho \\
& \leq \int_{-r}^{r} \frac{1+\rho}{1-\rho} \mathrm{d} \rho \\
& =2 \log (1+r)-2 r-2 \log (1-r)
\end{aligned}
$$

for all $\theta, 0 \leq \theta<2 \pi$. Therefore,

$$
l(r, f) \leq 2 \log (1+r)-2 r-2 \log (1-r) .
$$

This gives (2.8) and it completes the proof of Theorem. 2.4.

Let us define $M(r, f)$ by

$$
M(r, f)=\max _{0 \leq \theta<2 \pi}\left|f\left(r e^{i \theta}\right)\right| .
$$

Then F. R. Keogh [5] has shown that

2.5. Theorem. [5] Suppose that $f(z) \in \mathcal{S}^{*}$. Then we have

$$
L(r, f)=\mathcal{O}\left(M(r, f) \log \frac{1}{1-r}\right) \quad \text { as } \quad r \rightarrow 1,
$$

where $\mathcal{O}$ means Landau's symbol.

Furthermore, D. K. Thomas in [14] extended this result for bounded close-to-convex functions. A function $f(z)$ is said to be close-to-convex if $f \in \mathcal{A}$ satisfies

$$
\mathfrak{R e}\left\{\frac{z f^{\prime}(z)}{e^{i \alpha} g(z)}\right\}>0, z \in \mathbb{D}
$$

for some $g(z) \in \mathcal{S}^{*}$ and some $\alpha \in(-\pi / 2, \pi / 2)$. The class of close-to-convex functions is denoted by $\mathcal{K}$.

It is known that $\mathcal{K} \subset \mathcal{S}$. An univalent function $f \in \mathcal{S}$ belongs to $\mathcal{K}$ if and only if the complement $E$ of the image-region $F=\{f(z):|z|<1\}$ is the union of rays that are disjoint (except that the origin of one ray may lie on another one of the rays). 
Recall that $A(r, f)$ is the area enclosed by $C(r, f)$, where $C(r, f)$ is the image of $|z|=r<1$ under the mapping $w=f(z)$. In [15] D. K. Thomas has shown that

2.6. Theorem. [15, Th.1] If $f(z) \in \mathcal{S}^{*}$, then

$$
L(r, f) \leq 2 \sqrt{\pi A(r, f)}\left(1+\log \frac{1+r}{1-r}\right) \quad \text { as } \quad r \rightarrow 1 .
$$

Also, in [12] Ch. Pommerenke has shown that

2.7. Theorem. [12] If $f(z) \in \mathcal{K}$, then

$$
L(r, f)=\mathcal{O}\left\{M(r, f)\left(\log \frac{1}{1-r}\right)^{5 / 2}\right\} \quad \text { as } \quad r \rightarrow 1 .
$$

Moreover, in [8,9] the second author has shown that

2.8. Theorem. If $z f^{\prime}(z) \in \mathcal{S}^{*}$, then

$$
L(r, f)=\mathcal{O}\left\{A(r, f) \log \frac{1}{1-r}\right\}^{1 / 2} \quad \text { as } \quad r \rightarrow 1 .
$$

Here, we consider a related problem.

2.9. Theorem. Let $f(z)$ be of the form (1.1) and suppose that

$$
\left|1+\mathfrak{R e} \frac{z f^{\prime \prime}(z)}{f^{\prime}(z)}\right| \leq\left|\frac{1+z}{1-z}\right|^{\alpha} \quad z \in \mathbb{D},
$$

where $0<\alpha<1 / 2$. Then we have

$$
L(r, f)=\mathcal{O}\left(A(r, f) \log \frac{1}{1-r}\right)^{1 / 2} \quad \text { as } \quad r \rightarrow 1,
$$

where $\mathcal{O}$ means Landau's symbol.

Proof. It is known, for instance see [16, p.227], that if $h(z)=u(z)+i v(z)$ is analytic in $|z| \leq R$, then

$$
h(z)=\frac{1}{2 \pi} \int_{0}^{2 \pi} u\left(R e^{i \varphi}\right) \frac{R e^{i \varphi}+z}{R e^{i \varphi}-z} \mathrm{~d} \varphi+i v(0) .
$$

Moreover, if $|z|<R, v(0)=0$, then

$$
|h(z)| \leq \frac{1}{2 \pi} \int_{0}^{2 \pi}\left|u\left(R e^{i \varphi}\right)\right|\left|\frac{R e^{i \varphi}+z}{R e^{i \varphi}-z}\right| \mathrm{d} \varphi .
$$

Applying the harmonic function theory, Schwarz's lemma, Hayman's result [4, p.280] and [1, p.491]:

$$
\int_{0}^{2 \pi} \frac{\mathrm{d} \theta}{\left|1-r e^{i \theta}\right|^{\alpha}}= \begin{cases}\mathcal{O}\left((1-r)^{1-\alpha}\right) & \text { for the case } 1<\alpha, \\ \mathcal{O}\left(\log \frac{1}{1-r}\right) & \text { for the case } \alpha=1, \\ \mathcal{O}(1) & \text { for the case } 0 \leq \alpha<1\end{cases}
$$

where $0<r<1,0 \leq \theta \leq 2 \pi, 0 \leq \alpha$, and using the symbol $C$ to be an absolute positive constant, not necessary the same value at each time throughout in this paper, we have

$$
\begin{aligned}
L(r, f) & =\int_{0}^{2 \pi}\left|z f^{\prime}(z)\right| \mathrm{d} \theta \\
& \leq \int_{0}^{2 \pi} \int_{0}^{r}\left|f^{\prime}(z)+z f^{\prime \prime}(z)\right| \mathrm{d} \rho \mathrm{d} \theta \\
& =\int_{0}^{r} \int_{0}^{2 \pi}\left|f^{\prime}(z)\right|\left|1+\frac{z f^{\prime \prime}(z)}{f^{\prime}(z)}\right| \mathrm{d} \theta \mathrm{d} \rho .
\end{aligned}
$$


Therefore, we have

$$
\begin{aligned}
L(r, f) & \leq \frac{1}{2 \pi} \int_{0}^{r} \int_{0}^{2 \pi}\left|f^{\prime}(z)\right|\left(\int_{0}^{2 \pi}\left|1+\mathfrak{R e} \frac{\zeta f^{\prime \prime}(\zeta)}{f^{\prime}(\zeta)}\right|\left|\frac{\zeta+z}{\zeta-z}\right| \mathrm{d} \varphi\right) \mathrm{d} \theta \mathrm{d} \rho \\
& \leq \frac{1}{2 \pi} \int_{0}^{r} \int_{0}^{2 \pi}\left|f^{\prime}(z)\right|\left\{\int_{0}^{2 \pi}\left|\frac{1+\zeta}{1-\zeta}\right|^{2 \alpha} \mathrm{d} \varphi\right\}^{1 / 2}\left\{\int_{0}^{2 \pi}\left|\frac{\zeta+z}{\zeta-z}\right|^{2} \mathrm{~d} \varphi\right\}^{1 / 2} \mathrm{~d} \theta \mathrm{d} \rho \\
& \leq C \int_{0}^{r} \int_{0}^{2 \pi}\left|f^{\prime}(z)\right|\left(\frac{1}{|\zeta|^{2}-|z|^{2}}\right)^{1 / 2} \mathrm{~d} \theta \mathrm{d} \rho \\
& \leq C \int_{0}^{r} \int_{0}^{2 \pi}\left|f^{\prime}(z)\right|\left(\frac{1}{1-\rho^{2}}\right)^{1 / 2} \mathrm{~d} \theta \mathrm{d} \rho \\
& =C \int_{0}^{r} \int_{0}^{2 \pi}\left|\frac{f^{\prime}(z)}{\sqrt{1+\rho}}\right|\left(\frac{1}{1-\rho}\right)^{1 / 2} \mathrm{~d} \theta \mathrm{d} \rho,
\end{aligned}
$$

where $z=\rho e^{i \theta}, \zeta=|\zeta| e^{i \varphi}, 0 \leq|z|=\rho<|\zeta|<1,|\zeta|=\sqrt{\left(1+\rho^{2}\right) / 2}$ and where $0<r<1$. If $\rho \geq(\sqrt{5}-1) / 2$, then

$$
\frac{1}{1+\rho} \leq \rho
$$

Therefore, for $r_{1} \geq(\sqrt{5}-1) / 2$, we have

$$
\begin{aligned}
L(r, f) & \leq C\left(\int_{0}^{r} \int_{0}^{2 \pi} \frac{\left|f^{\prime}(z)\right|^{2}}{1+\rho} \mathrm{d} \theta \mathrm{d} \rho\right)^{1 / 2}\left(\int_{0}^{r} \int_{0}^{2 \pi} \frac{1}{1-\rho} \mathrm{d} \theta \mathrm{d} \rho\right)^{1 / 2} \\
& \leq C\left(\int_{0}^{r_{1}} \int_{0}^{2 \pi} \frac{\left|f^{\prime}(z)\right|^{2}}{1+\rho} \mathrm{d} \theta \mathrm{d} \rho+\int_{r_{1}}^{r} \int_{0}^{2 \pi} \rho\left|f^{\prime}(z)\right|^{2} \mathrm{~d} \theta \mathrm{d} \rho\right)^{1 / 2}\left(\log \frac{1}{1-r}\right)^{1 / 2} \\
& \leq C\left(\int_{0}^{r_{1}} \int_{0}^{2 \pi} \frac{\left|f^{\prime}(z)\right|^{2}}{1+\rho} \mathrm{d} \theta \mathrm{d} \rho+A(r, f)-A\left(r_{1}, f\right)\right)^{1 / 2}\left(\log \frac{1}{1-r}\right)^{1 / 2} .
\end{aligned}
$$

If $r \rightarrow 1$, we get (2.11).

Some length problems for analytic functions were considered also in our recent papers $[10,11]$.

\section{3. acknowledgement}

The authors express their sincerest thanks to the referees for some invaluable suggestions.

\section{References}

[1] P. Eenigenburg, On the radius of curvature for convex analytic functions, Canad. J. Math. $22(3)(1970) 486-491$.

[2] L. Fejér, F. Riesz, Über einige funktionentheoretische Ungleichungen, Math. Zeitschr. 11(1921) 305-314.

[3] A. W. Goodman, Univalent Functions, Vols. I and II, Mariner Publishing Co.: Tampa, Florida (1983).

[4] W. F. Hayman, The asymptotic behaviour of $p$-valent functions, Proc. London Math. Soc. $3(5)(1955)$ 257-284.

[5] F. R. Keogh, Some theorems on conformal mapping of bounded star-shaped domain, Proc. London Math. Soc. (3)9(1959) 481-491.

[6] M. Nunokawa, On the Univalency and Multivalency of Certain Analytic Functions, Math. Zeitschr. 104(1968) 394-404. 
[7] M. Nunokawa, S. Owa, S. Fukui, H. Saitoh, M.-P. Chen, A class of functions which do not assume non-positive real part, Tamkang J. Math. 19(2)(1968) 23-26.

[8] M. Nunokawa, On Bazilevič and convex functions, Trans. Amer. Math. Soc., 143(1969) $337-341$.

[9] M. Nunokawa, A note on convex and Bazilevič functions, Proc. Amer. Math. Soc., $24(2)(1970) 332-335$.

[10] M. Nunokawa, J. Sokól, On some length problems for analytic functions, Osaka J. Math. 51(2014) 695-707.

[11] M. Nunokawa, J. Sokól, On some length problems for univalent functions, Math. Meth. Appl. Sci., 39(7)(2016) 1662-1666.

[12] Ch. Pommerenke, Über nahezu konvexe analytische Functionen, Arch. Math. (Basel) 16(1965) 344-347.

[13] E. Study, Konforme Abbildung Einfachzusammenhangender Bereiche, B. C. Teubner, Leipzig und Berlin 1913.

[14] D. K. Thomas, On starlike and close-to-convex univalent functions, J. London Math. Soc. 42(1967) 427-435.

[15] D. K. Thomas, A note on starlike functions, J. London Math. Soc. 43(1968) 703-706.

[16] M. Tsuji, Complex Functions Theory, Maki Book Comp., Tokyo 1968.(Japanese) 
